\title{
AVALIAÇÃO DA AUTO PERCEPÇÃO DA SAÚDE BUCAL DAS GESTANTES: REVISÃO DE LITERATURA
}

\section{SELF-ASSESSMENT PERCEPTION OF ORAL HEALTH OF PREGNANT WOMEN: LITERATURE REVIEW}

Nilton Hiroji Akabane', Valéria Silva Candido Brizon (in Memorian), Antonio Carlos Pereira², Edna Zakzevski Padilha ${ }^{3}$, Fabrício Rutz da Silva ${ }^{3 *}$

'Prefeitura de Monte Mor, Monte Mor, São Paulo, Brasil, 2FOP/UNICAMP, Departamento de Odontologia Social, Piracicaba, São Paulo, Brasil, ' ${ }^{F}$ ASIPE, Departamento de Odontologia, Sinop, Mato Grosso, Brasil

"Autor correspondente: Avenida das Artes, quadra 26, lote 21, apto 13, CEP 78.555-474, Aquarela das Artes, Sinop, Mato Grosso. Telefone (42) 99960-7965. E-mail: fabriciorutz@hotmail.com

\section{RESUMO}

O objetivo do estudo foi avaliar a auto percepção de saúde bucal das gestantes. Foi realizada uma revisão de literatura sobre a auto percepção de saúde bucal das gestantes, com busca ativa nos banco de dados do Sistema Online de Busca e Análise de literatura médica (Medical Literature Analysis and Retrieval System Online-Medline), Pubmed, Centro Latino Americano e do Caribe de informação em Ciências da Saúde (BIREME), Literatura Latino Americana e do Caribe em Ciências da Saúde (LILACS) e Google Acadêmico, entre os anos de 2005 a 2015, com os descritores: gestante, auto percepção, saúde bucal, oral health, pregnant e selfreported, self-perception. Foram identificados 701 artigos sendo selecionados 14 através da leitura dos resumos em que o conteúdo discorria sobre a auto percepção da saúde bucal das gestantes. Concluiu-se que a auto percepção das gestantes é baixa em relação aos problemas de saúde bucal, indicando que a falta de conhecimento sobre o assunto faz com que as gestantes não identifiquem a necessidade da procura por tratamento odontológico. Programas educacionais devem ser criados a fim de oferecer mais conhecimento para esse grupo, além de incluir atendimento odontológico no pré-natal.

Descritores: gestantes, saúde bucal, auto percepção.

\section{ABSTRACT}

The objective of the study was to evaluate the self-perception of oral health of pregnant women. A review of the literature on the self-perception of oral health of pregnant women was carried out, with an active search in the databases of the Medical Literature Analysis and Retrieval System Online-Medline, Pubmed, Latin American Center (BIREME), Latin American and Caribbean Literature in Health Sciences (LILACS) and Google Academic, between 2005 and 2015, with the descriptors: pregnant women, self-perception, oral health, oral health, pregnant and self-reported, self-perception. A total of 701 articles were identified 14 by reading the abstracts in which the content was about the self-perception of the oral health of pregnant women. It was concluded that the self-perception of pregnant women is low in relation to oral health problems, indicating that the lack of knowledge about the subject makes the pregnant women do not identify the need for dental treatment. Educational programs should be created in order to offer more knowledge to this group, in addition to including prenatal dental care.

Descriptors: pregnant women, oral health, self-concept. 


\section{INTRODUÇÃO}

Durante a gravidez, ocorrem numerosas modificações fisiológicas em todo o corpo da mulher, principalmente nos sistemas respiratório, renal, gastrointestinal, endócrino e gênito-urinário (GAJENDRA; KUMAR, 2004; SURESH, L.; RADFAR, 2004). Dentre elas estão: aumento da salivação, náuseas e vômitos, ganho de peso exagerado, hipotensão postural, hipoglicemia, aumento da urina, restrição da função respiratória, síncopes e alterações nos batimentos cardíacos (SILVA; STUANI; QUEIROZ, 2006).

Na cavidade bucal, o aumento dos níveis de estrógeno e progesterona causa a hipervascularização do periodonto e alterações na produção do colágeno, além do aumento da permeabilidade capilar. Tal situação torna as respostas inflamatórias do tecido periodontal mais exacerbadas diante dos irritantes locais (BARAK; OETTINGER-BARAK; MACHTEI; 2003). Ocorre ainda, a redução da concentração de cálcio e fosfato na composição da saliva e a redução do pH salivar (SALVOLINI; GIORGIO; CURATOLA, 1998).

Na gestação, ocorrem mudanças de hábitos de dieta e higiene bucal que podem ser causadas pela diminuição da capacidade estomacal devido ao aumento do volume uterino, resultando em aumento da quantidade e frequência de ingestão de alimentos (POZO, 2001).

Os problemas bucais comumente encontrados na cavidade bucal da gestante são: doença periodontal, cárie, tumor gravídico dentre outras, sendo a gengivite, a patologia mais prevalente (35 a 100\% das gestantes) (PASSINI; NOMURA; POLITANO; 2007; HOM et al. 2012).

As doenças periodontais possuem efeitos nocivos tanto na gestante como no feto em desenvolvimento, pois a presença de infecções bacterianas estimula a produção de mediadores inflamatórios (prostaglandinas), os quais podem provocar o parto prematuro e baixo peso ao nascer (OFFENBACHER; KATZ; FERTIK, 1996; BOGGESS; EDELSTEIN, 2006; XIONG; BUEKENS; FRASER, 2006). Além disso, a doença periodontal também está relacionada ao aumento do risco da pré-eclâmpsia.

O nascimento prematuro é a principal causa de morbidade neonatal e mortalidade no mundo (WHO, 1977). Classicamente definido como o nascimento que ocorre antes da $37^{\mathrm{a}}$ semana de gravidez, ele também é o principal responsável por deficiências adquiridas após o nascimento (WHO, 1977). Exceto por malformações congênitas, 75\% das mortes perinatais e 50\% de anormalidades neurológicas estão diretamente atribuídas à condição de prematuridade (MARLOW; WOLKE; BRACEWELL, 2005).

A taxa de mortalidade infantil é medida pela frequência de óbitos de crianças até 1 ano de idade por mil nascidos vivos. Mais de $85 \%$ das mortes de crianças com menos de 5 anos no Brasil ocorre no primeiro ano de vida (BRASIL, 2014).

Para analisar o nível de desenvolvimento humano de um país, é preciso realizar estudos acerca de diversos indicadores sociais, mais especificamente sobre a condição de saúde, renda, educação e expectativa de vida (INSTITUTO BRASILEIRO DE GEOGRAFIA E ESTATÍSTICA, 2010).

Em 2000, os líderes mundiais se uniram e estabeleceram uma agenda global de compromissos mínimos pela promoção da dignidade humana, surgindo assim as metas do milênio ou os oito Objetivos de Desenvolvimento do Milênio (BRASIL, 2014).

Uma das metas ou o quarto Objetivo do Milênio (ODM 4) é a redução da mortalidade na infância, que leva em conta três indicadores: a taxa de mortalidade na infância (menores de 5 anos), a taxa de mortalidade infantil (menores de $1 \mathrm{ano}$ ) e a proporção de crianças de até 1 ano vacinadas contra o sarampo (BRASIL, 2014). 
Alguns programas do governo brasileiro têm o objetivo de reduzir a mortalidade materna e neonatal como é o caso da rede cegonha, lançada em 2011, baseada no pré-natal, parto, nascimento, puerpério e atenção à saúde da criança e sistema logístico (BRASIL, 2014).

Em gestantes de baixa escolaridade e renda, os problemas dentários são mais evidentes (YALCIN; ESKINAZI; SOYDINC, 2002; ZANATA et al., 2003; OLIVEIRA; NADANOVSKY, 2006). O alto custo e a dificuldade de acesso ao tratamento odontológico, além das crenças culturais, prejudicam a procura por atendimento (YALCIN; ESKINAZI; SOYDINC, 2002; ZANATA et al., 2003; OLIVEIRA; NADANOVSKY, 2006).

A importância de se entender a auto percepção da saúde bucal do indivíduo, é o fato de seus comportamentos biológicos, psicossociais e sociais serem condicionados por tal percepção (JYLHÄ et al., 1998). É também responsável pela motivação do paciente a ter consciência de sua própria condição, fazendo com que o interesse em se cuidar transforme-se em qualidade de vida (JEREMIAS et al., 2010).

Uma abordagem educativa, bem como a remoção do biofilme dental e eliminação de focos de infecção são medidas básicas que podem melhorar a qualidade de vida da gestante e do bebê (21).

\section{MATERIAIS E MÉTODO}

Foi realizada uma busca ativa nas bases de dados do Sistema Online de Busca e Análise de literatura médica (Medical Literature Analysis and Retrieval System Online-Medline), Pubmed, Centro Latino Americano e do Caribe de informação em Ciências da Saúde (BIREME), Literatura Latino Americana e do Caribe em Ciências da Saúde (LILACS) e Google Acadêmico, utilizando os seguintes descritores: auto percepção, gestante, saúde bucal, self-perception, pregnant, oral health e self-reported. A busca abrangeu artigos publicados nos últimos 10 anos em qualquer idioma. Como critério de exclusão, foram desconsiderados aqueles que não abordavam o assunto delimitado: auto percepção de saúde bucal da gestante.

\section{RESULTADOS}

Foram identificados 701 artigos, sendo selecionados 14 através da leitura dos títulos e resumos em que o conteúdo discorria sobre a auto percepção de saúde bucal da gestante. Esses trabalhos foram lidos na íntegra e estão listados no quadro I.

O Quadro I mostra os artigos distribuídos de acordo com o título, autores, ano de publicação, público alvo, número da amostra e o tipo de estudo/método. 
Tabela 1 - Características dos estudos sobre a auto percepção da saúde bucal das gestantes, segundo o título, autores, ano de publicação, público alvo, número da amostra e o tipo de estudo/método

\begin{tabular}{|c|c|c|c|c|c|}
\hline Título do artigo & Autores & $\begin{array}{c}\text { Ano de } \\
\text { publicação }\end{array}$ & Público alvo & $\begin{array}{l}\mathrm{N}^{0} \text { da } \\
\text { amostra }\end{array}$ & Tipo de estudo \\
\hline $\begin{array}{l}\text { Self-perception and } \\
\text { Oral Health in Pregnant } \\
\text { Adolescents }\end{array}$ & $\begin{array}{l}\text { Saliba Rovida TA, } \\
\text { Saliba Moimaz SA, } \\
\text { Lima DP, Saliba } \\
\text { Garbin CA }\end{array}$ & 2014 & Adolescentes grávidas & $\begin{array}{l}127 \\
\text { adolescentes }\end{array}$ & $\begin{array}{l}\text { Estudo } \\
\text { transversal } \\
\text { tipo survey e } \\
\text { pesquisa de } \\
\text { condições de } \\
\text { saúde bucal } \\
\end{array}$ \\
\hline $\begin{array}{l}\text { Percepção das gestantes } \\
\text { do Programa de Saúde da } \\
\text { Família em relação à saúde } \\
\text { bucal no município de } \\
\text { Ribeirópolis, Sergipe, Brasil }\end{array}$ & $\begin{array}{l}\text { Marlos Cesar } \\
\text { Bomfim Cabral, } \\
\text { Thiago de Santana } \\
\text { Santos, Thiago } \\
\text { Pelúcio Moreira } \\
\end{array}$ & 2013 & $\begin{array}{l}\text { Mulheres grávidas } \\
\text { cadastradas no } \\
\text { programa de pré natal } \\
\text { (SIS Pré Natal) no mês de } \\
\text { fevereiro de } 2010 .\end{array}$ & $\begin{array}{l}41 \\
\text { gestantes }\end{array}$ & $\begin{array}{l}\text { Qualitativa e } \\
\text { quantitativa }\end{array}$ \\
\hline $\begin{array}{l}\text { A Saúde Bucal de uma } \\
\text { Subpopulação de Gestantes } \\
\text { Usuárias do Sistema Único de } \\
\text { Saúde: um Estudo Piloto }\end{array}$ & $\begin{array}{l}\text { Débora Prado } \\
\text { Martins, Álvaro } \\
\text { Henrique Borges, } \\
\text { Alex Semenoff } \\
\text { Segundo, Vinícius } \\
\text { Canavarros Palma, } \\
\text { Luiz Evaristo Ricci } \\
\text { Volpato } \\
\end{array}$ & 2013 & $\begin{array}{l}\text { Puérperas internadas } \\
\text { em hospital do SUS em } \\
\text { Cuiabá, Mato Grosso, } \\
\text { em } 2011 .\end{array}$ & $\begin{array}{l}121 \\
\text { puérperas }\end{array}$ & $\begin{array}{l}\text { Transversal } \\
\text { descritivo }\end{array}$ \\
\hline $\begin{array}{l}\text { Self-reported oral health, oral } \\
\text { hygiene habits and dental } \\
\text { service utilization among } \\
\text { pregnant women in United } \\
\text { Arab Emirates }\end{array}$ & Hashim R & 2012 & $\begin{array}{l}\text { Gestantes que } \\
\text { frequentavam as } \\
\text { maternidades de Dubai, } \\
\text { Sharja e Ajman no } \\
\text { qual foram escolhidas } \\
\text { por programa de } \\
\text { computador de forma } \\
\text { randomizada. }\end{array}$ & $\begin{array}{l}800 \\
\text { gestantes }\end{array}$ & $\begin{array}{l}\text { Corte } \\
\text { transversal }\end{array}$ \\
\hline $\begin{array}{l}\text { Percepção de primigestas } \\
\text { adolescentes sobre saúde } \\
\text { bucal }\end{array}$ & $\begin{array}{l}\text { Bruna Ferreira } \\
\text { Amorim, José } \\
\text { Ferreira Costa, } \\
\text { Elizabeth Lima } \\
\text { Costa }\end{array}$ & 2011 & $\begin{array}{l}\text { Gestantes inscritas no } \\
\text { Programa de Pré-natal } \\
\text { do Hospital Maternidade } \\
\text { Marly Sarney, São Luís, } \\
\text { MA, em } 2011 \text {. }\end{array}$ & $\begin{array}{l}40 \\
\text { gestantes. }\end{array}$ & $\begin{array}{l}\text { Estudo } \\
\text { transversal } \\
\text { através de } \\
\text { questionário } \\
\text { estruturado } \\
\text { com questões } \\
\text { específicas } \\
\text { sobre saúde } \\
\text { bucal. } \\
\end{array}$ \\
\hline $\begin{array}{l}\text { Self-reported oral health and } \\
\text { hygiene habits, dental decay } \\
\text { and periodontal condition } \\
\text { among pregnant European } \\
\text { women }\end{array}$ & $\begin{array}{l}\text { Yolanda Martínez- } \\
\text { Beneyto, María } \\
\text { V. Vera-Delgado, } \\
\text { Leonor Pérez, } \\
\text { Antonio Maurandi } \\
\end{array}$ & 2011 & $\begin{array}{l}\text { Mulheres grávidas } \\
\text { vivendo em Murcia, } \\
\text { sudeste da Espanha. }\end{array}$ & $\begin{array}{l}337 \\
\text { gestantes }\end{array}$ & $\begin{array}{l}\text { Estudo } \\
\text { transversal }\end{array}$ \\
\hline $\begin{array}{l}\text { Oral health conditions among } \\
\text { pregnant women attended } \\
\text { to at a health care center in } \\
\text { Manaus, Amazonas, Brazil }\end{array}$ & $\begin{array}{l}\text { Larissa Borges } \\
\text { Bressane, Lívea } \\
\text { Nancy Bulcão da } \\
\text { Silva Costa, Janete } \\
\text { Maria Rebelo } \\
\text { Vieira, Maria } \\
\text { Augusta Bessa } \\
\text { Rebelo }\end{array}$ & 2011 & $\begin{array}{l}\text { Gestantes que } \\
\text { frequentam o programa } \\
\text { de pré natal na unidade } \\
\text { básica de saúde de } \\
\text { Theomário Pinto Cost, } \\
\text { localizado na região } \\
\text { centro sul de Manaus, } \\
\text { Amazonas. }\end{array}$ & $\begin{array}{l}50 \\
\text { gestantes }\end{array}$ & $\begin{array}{l}\text { Descritivo } \\
\text { e corte } \\
\text { transversal }\end{array}$ \\
\hline $\begin{array}{l}\text { Autopercepção e Condições } \\
\text { de Saúde Bucal em } \\
\text { Gestantes }\end{array}$ & $\begin{array}{l}\text { Fabiano Jeremias, } \\
\text { Silvio Rocha Corrêa } \\
\text { da Silva, Aylton } \\
\text { Valsecki Junior, } \\
\text { Elaine Pereira da } \\
\text { Silva Tagliaferro, } \\
\text { Fernanda Lopez } \\
\text { Rosell }\end{array}$ & 2010 & $\begin{array}{l}\text { Gestantes } \\
\text { frequentadoras da } \\
\text { Clínica de Odontologia } \\
\text { Preventiva e Sanitária da } \\
\text { FoAr-Unesp, em } 2008 .\end{array}$ & $\begin{array}{l}50 \\
\text { gestantes }\end{array}$ & $\begin{array}{l}\text { Questionário } \\
\text { semi- } \\
\text { estruturado. }\end{array}$ \\
\hline
\end{tabular}




\begin{tabular}{|c|c|c|c|c|c|}
\hline Título do artigo & Autores & $\begin{array}{c}\text { Ano de } \\
\text { publicação }\end{array}$ & Público alvo & $\begin{array}{l}\mathrm{N}^{0} \mathrm{da} \\
\text { amostra }\end{array}$ & Tipo de estudo \\
\hline $\begin{array}{l}\text { Prevalência de cárie } \\
\text { em adolescentes } \\
\text { gestantes relacionada ao } \\
\text { conhecimento sobre saúde } \\
\text { bucal em Porto Velho-RO }\end{array}$ & $\begin{array}{l}\text { Cleson Oliveira de } \\
\text { Moura, Rodrigo } \\
\text { Queiroz Aleixo, } \\
\text { Frank Aragão de } \\
\text { Almeida, Henriete } \\
\text { Mac-Lins Lima } \\
\text { e Silva, Kátia } \\
\text { Fernanda Alves } \\
\text { Moreira }\end{array}$ & 2010 & $\begin{array}{l}\text { Gestantes adolescentes } \\
\text { de uma comunidade de } \\
\text { Porto Alegre, RO. }\end{array}$ & $\begin{array}{l}26 \\
\text { gestantes }\end{array}$ & $\begin{array}{l}\text { Pesquisa } \\
\text { transversal com } \\
\text { abordagem } \\
\text { quantitativa } \\
\text { através de } \\
\text { questionário } \\
\text { com questões } \\
\text { pré-elaboradas } \\
\text { e exame } \\
\text { clínico. }\end{array}$ \\
\hline $\begin{array}{l}\text { Percepção das gestantes } \\
\text { quanto a sua saúde bucal }\end{array}$ & $\begin{array}{l}\text { Graciene de } \\
\text { Fátima Vieira, Keli } \\
\text { Bahia Felicíssimo } \\
\text { Zocratto }\end{array}$ & 2007 & $\begin{array}{l}\text { Gestantes do município } \\
\text { de Belo Horizonte, MG, } \\
\text { em 2005, em locais de } \\
\text { grande concentração } \\
\text { de pessoas (ex: } \\
\text { supermercados, } \\
\text { shoppings) e aos redores } \\
\text { das UBS. }\end{array}$ & $\begin{array}{l}76 \\
\text { gestantes }\end{array}$ & $\begin{array}{l}\text { Descritivo } \\
\text { e desenho } \\
\text { transversal } \\
\text { através de } \\
\text { questionário } \\
\text { semi- } \\
\text { estruturado. }\end{array}$ \\
\hline $\begin{array}{l}\text { Percepção de gestantes } \\
\text { do Amazonas em relação à } \\
\text { saúde bucal. }\end{array}$ & $\begin{array}{l}\text { Savana de Alencar } \\
\text { Maia, Pollyana } \\
\text { Cinthia de Souza } \\
\text { da Silva, Maria } \\
\text { Eliana Cruz de } \\
\text { Almeida, Andrea } \\
\text { Melo Moutinho da } \\
\text { Costa. }\end{array}$ & 2007 & $\begin{array}{l}\text { Gestantes atendidas no } \\
\text { pré-natal do centro de } \\
\text { saúde da zona lesta de } \\
\text { Manaus/Amazonas }\end{array}$ & $\begin{array}{l}43 \\
\text { selecionadas } \\
\text { a partir } \\
\text { de } 200 \\
\text { gestantes }\end{array}$ & $\begin{array}{l}\text { Transversal } \\
\text { descritivo } \\
\text { através de } \\
\text { aplicação de } \\
\text { questionário. }\end{array}$ \\
\hline $\begin{array}{l}\text { Conhecimento de gestantes } \\
\text { sobre de saúde bucal no } \\
\text { município de Caruaru-PE }\end{array}$ & $\begin{array}{l}\text { Ana Flávia } \\
\text { Granville- } \\
\text { Garcia, Angélica } \\
\text { Falcão Leite, } \\
\text { Lydia Elizabeth } \\
\text { Ataíde Smith, } \\
\text { Renata Vale de } \\
\text { Siqueira Campos, } \\
\text { Valdenice } \\
\text { Aparecida de } \\
\text { Menezes. }\end{array}$ & 2007 & $\begin{array}{l}\text { Gestantes atendida } \\
\text { em duas instituições de } \\
\text { saúde pública e privada }\end{array}$ & $\begin{array}{l}100 \\
\text { gestantes }\end{array}$ & $\begin{array}{l}\text { Estudo } \\
\text { transversal }\end{array}$ \\
\hline $\begin{array}{l}\text { Percepção das condições de } \\
\text { saúde bucal por gestantes } \\
\text { atendidas em uma unidade } \\
\text { de saúde no município de } \\
\text { Araraquara, São Paulo, Brasil }\end{array}$ & $\begin{array}{l}\text { Silvio Rocha Corrêa } \\
\text { da Silva, Fernanda } \\
\text { Lopez Rosell, Aylton } \\
\text { Valsecki Júnior }\end{array}$ & 2006 & $\begin{array}{l}\text { Gestantes } \\
\text { frequentadoras de uma } \\
\text { unidade básica de } \\
\text { saúde de Araraquara, SP }\end{array}$ & $\begin{array}{l}53 \\
\text { gestantes }\end{array}$ & $\begin{array}{l}\text { Transversal } \\
\text { descritivo } \\
\text { através de } \\
\text { aplicação de } \\
\text { questionário. } \\
\end{array}$ \\
\hline $\begin{array}{l}\text { Self-reported oral health, oral } \\
\text { hygiene habits and dental } \\
\text { attendance of pregnant } \\
\text { women in Kuwait }\end{array}$ & $\begin{array}{l}\text { Sisko Honkala, } \\
\text { Jassem Al-Ansari }\end{array}$ & 2005 & $\begin{array}{l}\text { Gestantes internadas na } \\
\text { maternidade do governo } \\
\text { da cidade de Kuwait }\end{array}$ & $\begin{array}{l}603 \\
\text { gestantes }\end{array}$ & $\begin{array}{l}\text { Corte } \\
\text { transversal }\end{array}$ \\
\hline
\end{tabular}

Fonte: Os autores

\section{DISCUSSÃO}

As gestantes pertencem a um grupo de risco quando se refere aos problemas de saúde bucal como cáries e doenças gengivais, pois, associadas às alterações fisiológicas inerentes à fase gestacional, existem as mudanças comportamentais que são comuns neste período tais como: aumento da frequência de ingestão de alimentos, enjoos, vômitos e sonolência (BARAK et al. 2003).

A importância da auto percepção se deve ao fato da possibilidade do indivíduo ter consciência da própria condição de saúde, para assim ter uma mudança de comportamento devido à percepção da necessidade (CABRAL; SANTOS; MOREIRA, 2013). 
Foram encontrados seis artigos em que, além da pesquisa subjetiva sobre a auto percepção da saúde bucal das gestantes, foi abordado o lado quantitativo através do exame clínico bucal das voluntárias. Nos artigos de JEREMIAS et al (2010); SALIBA et al. (2014); MARTINS et al. (2013) e BRESSANE et al. (2011), os resultados demonstraram uma baixa auto percepção de saúde bucal pois a maioria das gestantes relatavam bom ou médio estado de saúde bucal, mas ao exame clínico foi constatado alto índice de cárie. Entretanto nos estudos de MOURA et al. (2010) e MARTINEZ-BENEYITO et al. (2011), as gestantes relataram uma auto percepção de acordo com o estado clínico examinado. Os outros oito artigos foram baseados em pesquisas feitas através de questionários. Desses, três artigos encontraram que as gestantes percebem a presença de problema bucal, mas não procuram o tratamento (HONKALA; AL-ANSARI, 2005; HASHIM, 2012; CABRAL; SANTOS; MOREIRA, 2013). Os demais artigos mostram que elas consideram o seu estado de saúde bucal bom, apesar de coexistir problemas bucais (SILVA; STUANI; QUEIROZ, 2006; VIEIRA; ZOCRATTO, 2007; MAIA; SILVA; ALMEIDA, 2007; GRANVILLE-GARCIA; LEITE; SMITH, 2007; AMORIM; COSTA; COSTA, 2011).

Nos estudos com gestantes adolescentes, a baixa escolaridade das entrevistadas além da maturidade física e emocional, associada ao fator social e falta de instrução para as modificações que estão sujeitas, tornam-nas vulneráveis na primeira gestação (AMORIM; COSTA; COSTA, 2011). Os estudos de MOURA et al. (2010) e MAIA et al. (2007), apresentaram resultados que corroboram com a afirmação.

ALBUQUERQUE, ABEGG e RODRIGUES (2004), citam barreiras ao tratamento odontológico durante a gravidez, tal como a baixa percepção da necessidade, o que foi comprovado nos estudos de BRESSANE et al. (2011), onde 78\% relatou ter saúde bucal boa ou média, mas 100\% das voluntárias apresentaram cárie. HONKALA \& AL-ANSARI (2005), 82\% relatavam ter saúde bucal boa ou muito boa, mas $30 \%$ sentiram que tinham problemas gengivais e $40 \%$ sentiram dor dental nos últimos seis meses, o que demonstra que as gestantes acreditam ser normal ter dor ou sangramento gengival durante a gestação.

O nível de escolaridade tem uma relação positiva com a auto percepção, ou seja, quanto maior o nível de escolaridade, melhor é a auto percepção. O que foi comprovado nos estudos de MARTINEZBENEYITO et al. (2011), em que 80,1\% tinham nível de escolaridade médio-alto e teve como conclusão que a auto percepção da saúde bucal era condizente com estado dental.

De acordo com BRAZ et al. (2010), são barreiras para a adesão ao tratamento mitos e crendices; tal afirmação corrobora os dados obtidos no estudo de VIEIRA \& ZOCRATTO (2010), onde 55,3\% das gestantes voluntárias, não foram ao dentista durante a gravidez e destas 21,4\% não foram porque acreditavam que grávidas não podiam receber atendimento odontológico. No trabalho de GRANVILLEGARCIA et al. (2007), 55,2\% das gestantes tinham medo de realizar tratamento odontológico por receio de prejudicar o bebê. Valor próximo foi encontrado no estudo de CABRAL et al. (2013), em que 63,4\% também acreditavam em contraindicação do tratamento odontológico. Na pesquisa de AMORIM et al. (2011), um outro mito foi relatado: 87,5\% das entrevistadas disseram que gravidez causa cáries.

As ações educativo-preventivas com gestantes qualificam sua saúde e tornam-se fundamentais para introduzir bons hábitos desde o inicio da vida da criança (2004). Tal afirmação nos norteia no sentido de que devemos orientar as gestantes sobre prevenção de doenças bucais, mas os artigos de HONKALA; AL-ANSARI, (2005); AMORIM et al. (2011); CABRAL et al. (2013); MARTINS et al. (2013), nos mostram que poucas gestantes recebem orientação de saúde bucal, $24,7 \%, 22 \%$, 31,7\% e 30\% respectivamente, denotando que um percentual pequeno está sendo orientada, porém no estudo de BRESSANE et al. (2011), 60,4\% das gestantes receberam orientação de saúde bucal. 


\section{CONCLUSÃO}

A auto percepção das gestantes mostrou-se baixa, em relação à saúde bucal, o que identifica que a falta de conhecimento faz com que não haja a percepção da necessidade. A baixa escolaridade ainda é uma barreira a ser vencida, para possibilitar o empoderamento das gestantes em relação ao seu corpo e saúde. Mitos e crenças relacionados à gestação ainda se encontram arraigados na cultura da população, levando esse grupo a não buscar atendimento odontológico.

Portanto, programas educacionais deveriam ser criados a fim de oferecer mais conhecimento para as gestantes, incluindo o atendimento odontológico no pré-natal, fortalecendo a necessidade da atuação do cirurgião-dentista neste período tão significativo da vida das mulheres.

\section{REFERÊNCIAS}

1. AlBUQUerque, O. M. R.; ABEGG, C.; RODRIGUES, C. S. Percepção das gestantes do Programa Saúde da Família em relação as barreiras no atendimento odontológico em Pernambuco, Brasil. Cadernos de Saúde Pública, v. 20, n. 3, p. 789-796, 2004.

2. AMORIM, B. F.; COSTA, J. F.; COSTA, E. L. Percepção de primigestas adolescentes sobre saúde bucal. Revista de Pesquisa Saúde, v. 12, n. 2, p. 13-17, 2011.

3. BARAK, S.; OETTINGER-BARAK, O. O. M.; MACHTEl, E. E. et al. Common oral manifestations during pregnancy: a review. Obstetrical \& Gynecological Survey, v. 58, n. 9, p. 624-628, 2003.

4. BOGGESS, K. A.; EDELSTEIN, B. L. Oral health in women during preconception and pregnancy: implications for birth outcomes and infant oral health. Maternal and Child Health Journal, v. 10, suppl 5, p. S169-S174, 2006.

5. BRASIL. Instituto de Pesquisa Econômica Aplicada. Objetivos de Desenvolvimento do Milênio: Relatório Nacional de Acompanhamento. Brasília, 2014.

6. BRASIL. Ministério da Saúde. Programa de humanização do Parto: Humanização no Pré-natal e Nascimento. Brasília, 2004.

7. BRÁZ, G.; MACHADO, F. C.; OLIVEIRA, A. S. et al. A experiência de um programa de atenção a saúde bucal no atendimento a gestantes. HU Revista, v. 36, n. 4, p. 324-332, 2010.

8. BRESSANE, L. B.; COSTA, L. N. B. S.; VIEIRA, J. M. R. et al. Oral health conditions among pregnant women attended to at a health care center in Manaus, Amazonas, Brazil. Revista Odonto Ciência, v. 26, n. 4, p. 291-296, 2011.

9. CABRAL, C. B. C.; SANTOS, T. S.; MOREIRA, T. P. Percepção das gestantes do Programa de Saúde da Família em relação à saúde bucal no município de Ribeirópolis, Sergipe, Brasil. Revista Portuguesa de Saúde Pública, v. 31, n. 2, p. 173-180, 2013.

10. GAJENDRA, S.; KUMAR, J. V. Oral Health and Pregnancy: A review. The New York State Dental. Journal, v. 70, n. 1, p. 40-44, 2004.

11. GRANVILLE-GARCIA, A. F.; LEITE, A. F.; SMITH, L. E. A. et al. Conhecimento de gestantes sobre saúde bucal no município de Caruaru-PE. Revista de Odontologia da UNESP, 2007; 36(3): 243249.

12. HASHIM. R. Self-reported oral health, oral hygiene habits and dental service utilization among pregnant women in United Arab Emirates. International Journal of Dental Hygiene, v. 10, n. 10, p. 142-146, 2012. 
13. HOM, J. M.; LEE, J. Y.; DIVARIS, K. et al. Oral health literacy and knowledge among patients who are pregnant for the first time. Journal of American Dental Association, v. 143, n. 9, p. 972-980, 2012.

14. HONKALA, S.; AL-ANSARI, J. Self-reported oral health, oral hygiene habits, and dental attendance of pregnant women in Kuwait. Journal Clinical Periodontology, v. 32, p. 809-814, 2005.

15. INSTITUTO BRASILEIRO DE GEOGRAFIA E ESTATÍSTICA. Síntese de Indicadores Sociais. uma análise das condições de vida da população brasileira, 2010. Disponível em: http://www.ibge.gov.br/ home/estatistica/populacao/condicaodevida/indicadoresminimos/sinteseindicsociais2010/ SIS_2010.pdf. Acesso em: 05/06/2015.

16. JEFFCOAT, M. K.; HAULTH, J. C.; GEURS, N. C. et al. Periodontal disease and preterm birth: results of a pilot intervention study. Journal of Periodontology, v. 74, n. 8, p. 1214-1218, 2003.

17. JEREMIAS, F.; SILVA, S. R. C.; VALSECKI, JR. A. et al. Auto percepção e condições de saúde bucal em gestantes. Odontologia Clínico-Científica, v. 9, n. 4, p. 359-363, 2010.

18. JYLHÄ, M.; GURALNIK, J. M.; FERRUCCI, L. et al. Is self-rated health comparable across cultures and genders? The Journals of Gerontology. Series B, Psychological Sciences and Social Sciences, v. 53, n. 3, p. 144-152, 1998.

19. MAIA, A. S.; SILVA, P. C. S.; ALMEIDA, M. E. C. et al. Percepção de gestantes do Amazonas em relação à saúde bucal. ConScientiae Saúde, v. 6, n. 2, p. 377-383, 2007.

20. MARLOW, N.; WOLKE, D.; BRACEWELL, M. A. Neurologic and developmental disability at six years of age after extremely preterm birth. The New England Journal of Medicine, v. 352, n. 1, p. 9-19, 2005.

21. MARTíNEZ-BENEYTO, Y.; VERA-DELGADO, M. V.; PÉREZ, L. et al. Self-reported oral health and hygiene habits, dental decay, and periodontal condition among pregnant European women. International Journal of Gynecology and Obstetrics, v. 114, n.1, p. 18-22, 2011.

22. MARTINS, D. P.; BORGES, A. H.; SEGUNDO, A. S. et al. A Saúde Bucal de uma Subpopulação de Gestantes Usuárias do Sistema Único de Saúde: um Estudo Piloto. Pesquisa Brasileira em Odontopediatria e Clínica Integrada, v. 13, n. 3, p. 273-278, 2013.

23. MOURA, C. O.; ALEIXO, R. Q.; ALMEIDA, F.A. et al. Prevalência de cárie em adolescentes gestantes relacionada ao conhecimento sobre saúde bucal em Porto Velho-RO. Saber Científico Odontológico, v. 1, n. 1 p. 01-20, 2010.

24. OFFENBACHER, S.; KATZ, V.; FERTIK, G. et al. The periodontal infection as a possible risk factor for preterm low birth weight. Journal of Periodontology, v. 67, suppl 10, p.1103-1113, 1996.

25. OLIVEIRA, B. H.; NADANOVSKY, P. The impact of oral pain on quality of life during pregnancy in low-income Brazilian women. Journal of Orofacial Pain, v. 20, n. 4, p. 297-305, 2006.

26. PASSINI, JR. R.; NOMURA, M. L.; POLITANO, G. T. Doença periodontal e complicações obstétricas: há relação de risco? Revista Brasileira de Ginecologia e Obstetrícia, v. 29, n. 7, p. 370-375, 2007.

27. POZO, M. A. P. Tratamiento dental de la paciente gestante. Mundo odontológico, v. 8, n. 4, p. 54-59, 2001. 
28. SALIBA ROVIDA, T. A.; SALIBA MOIMAZ, A. S.; LIMA, D. P. et al. Self-perception and Oral Health in Pregnant Adolescents. Oral Health and Dental Management, v. 13, n. 3, p. 842-846, 2014.

29. SALVOLINI, E.; GIORGIO, R.; CURATOLA, A. et al. Biochemical modifications of human whole saliva induced by pregnancy. British Journal of Obstetrics Gynaecologic, v. 105, n, 6, p. 656-660, 1998.

30. SILVA, F. W. G. P.; STUANI, A. S.; QUEIROZ, A. M. Atendimento Odontológico à gestante. Parte 2: Cuidados durante a consulta. Revista Faculdade de Odontologia de Porto Alegre, v. 47, n. 3, p. $5-9,2006$.

31. SURESH, L.; RADFAR L. Pregnancy and lactation. Oral Surgery. Oral Medicine, Oral Pathology, Oral Radiology, and Endodontics, v. 97, n. 6, p. 672-682, 2004.

32. SILVA, S. R. C.; ROSELL, F. L.; VALSECKI JÚNIOR, A. Percepção das condições de saúde bucal por gestantes atendidas em uma unidade de saúde no município de Araraquara, São Paulo, Brasil. Revista. Brasileira de Saúde Materna Infantil, v. 6, n. 4, p. 405-410, 2006.

33. VIEIRA, G. F.; ZOCRATTO, K. B. F. Percepção das gestantes quanto a sua saúde bucal. Revista da Faculdade de Odontologia de Passo Fundo, v. 12, n. 2, p. 27-31, 2007.

34. WHO: recommended definitions, terminology and format for statistical tables related to the perinatal period and use of a new certificate for cause perinatal deaths. Modifications recommended by FIGO as amended October 14, 1976. Acta Obstetricia et Gynecologica Scandinavica, v. 56, n. 3, p. 247-253, 1977.

35. XIONG, X.; BUEKENS, P.; FRASER, W. D. Periodontal disease and adverse pregnancy outcomes: a systematic review. BJOG: an international journal of obstetrics and gynaecology, v. 113, n. 2, p. 135-143, 2006.

36. YALCIN, F.; ESKINAZI, E.; SOYDINC, M. et al. The effect of sociocultural status on periodontal condition in pregnancy. Journal of Periodontology, v. 73, n. 2, p. 178-182, 2002.

37. ZANATA, R. L.; NAVARRO, M. F. L.; PEREIRA, J. C. et al. Effect of caries preventive measures directed to expectant mothers on caries experience in their children. Brazilian Dental Journal, $v$. 14, n. 2, p. 75-81, 2003. 